\title{
Non-Celiac Wheat Sensitivity Diagnosed by Double-Blind Placebo-Controlled Challenge: Exploring a New Clinical Entity
}

\author{
Antonio Carroccio, MD¹, Pasquale Mansueto, MD², Giuseppe lacono, MD³, Maurizio Soresi, MD², Alberto D’Alcamo, MD², \\ Francesca Cavataio, MD³ , Ignazio Brusca, MD4, Ada M. Florena, MD5, Giuseppe Ambrosiano, MD², Aurelio Seidita, MD², \\ Giuseppe Pirrone, $\mathrm{MD}^{2}$ and Giovanni Battista Rini, MD²
}

OBJECTIVES: Non-celiac wheat sensitivity (WS) is considered a new clinical entity. An increasing percentage of the general population avoids gluten ingestion. However, the real existence of this condition is debated and specific markers are lacking. Our aim was thus to demonstrate the existence of WS and define its clinical, serologic, and histological markers.

METHODS: $\quad$ We reviewed the clinical charts of all subjects with an irritable bowel syndrome (IBS)-like presentation who had been diagnosed with WS using a double-blind placebo-controlled (DBPC) challenge in the years 2001-2011. One hundred celiac disease (CD) patients and fifty IBS patients served as controls.

RESULTS: $\quad$ Two hundred and seventy-six patients with WS, as diagnosed by DBPC challenge, were included. Two groups showing distinct clinical characteristics were identified: WS alone (group 1) and WS associated with multiple food hypersensitivity (group 2). As a whole group, the WS patients showed a higher frequency of anemia, weight loss, self-reported wheat intolerance, coexistent atopy, and food allergy in infancy than the IBS controls. There was also a higher frequency of positive serum assays for IgG/lgA anti-gliadin and cytometric basophil activation in "in vitro" assay. The main histology characteristic of WS patients was eosinophil infiltration of the duodenal and colon mucosa. Patients with WS alone were characterized by clinical features very similar to those found in CD patients. Patients with multiple food sensitivity were characterized by clinical features similar to those found in allergic patients.

CONCLUSIONS: Our data confirm the existence of non-celiac WS as a distinct clinical condition. We also suggest the existence of two distinct populations of subjects with WS: one with characteristics more similar to $C D$ and the other with characteristics pointing to food allergy.

SUPPLEMENTARY MATERIAL is linked to the online version of the paper at http://www.nature.com/ajg

Am J Gastroenterol 2012; 107:1898-1906; doi:10.1038/ajg.2012.236; published online 24 July 2012

\section{INTRODUCTION}

Celiac disease (CD) prevalence is estimated to be near to 1:100 in Western countries (1-3). However, a much higher percentage of the general population than this $1 \%$ consider themselves to be suffering from wheat sensitivity (WS) and exclude wheat from their diet on the basis of their negative experience after eating wheat-containing foods. These wheat-reactive patients often present symptoms similar to $\mathrm{CD}$ but have negative $\mathrm{CD}$ serology and histopathology.
In most cases, they consult a number of physicians, seeking to "reach" a diagnosis of $\mathrm{CD}$ but, very often, they are considered to be "simply" suffering from irritable bowel syndrome (IBS) (4). The diagnostic "gold standard" for WS ought to be the double-blind placebocontrolled (DBPC) challenge, but this is a quite cumbersome and time-consuming method and is therefore very rarely used.

In this retrospective study, we report the characteristics of all the patients with an IBS-like clinical presentation, who had received a

${ }^{1}$ Division of Internal Medicine, Hospital of Sciacca, ASP, Agrigento, Italy; ${ }^{2}$ Division of Internal Medicine, Policlinico University Hospital of Palermo, Palermo, Italy; ' ${ }^{3}$ ivision of Pediatric Gastroenterology, "Di Cristina" Hospital, Palermo, Italy; ${ }^{4}$ Department of Clinical Chemistry, Buccheri-La Ferla Hospital, Palermo, Italy; ${ }^{5}$ Department of Pathology, Policlinico University Hospital of Palermo, Palermo, Italy. Correspondence: Antonio Carroccio, MD, Medicina Interna, Hospital of Sciacca, ASP, Agrigento, Italy. E-mail: acarroccio@hotmail.com or pasquale.mansueto@unipa.it

Received 25 December 2011; accepted 26 June 2012 
definitive diagnosis of WS on the basis of a DBPC challenge in our centers in previous years.

Aims of the study were: (i) to demonstrate the existence of nonceliac WS as a defined clinical condition and (ii) to identify the clinical, serological, and histological characteristics, which could distinguish patients with non-CD WS from IBS and from CD patients.

\section{METHODS}

We reviewed the clinical charts of all patients with an IBS-like clinical presentation attending the outpatient center of the Department of Internal Medicine at the University Hospital of Palermo and the Department of Internal Medicine of the Hospital of Sciacca who had been diagnosed with WS between January 2001 and June 2011.

\section{Inclusion and exclusion criteria}

We included only patients who fulfilled the criteria recently proposed by the experts' meeting on "gluten sensitivity" $(5,6)$. In brief, the patients met all the following inclusion criteria:

(i) IBS-like symptoms defined according to the Rome II criteria (7).

(ii) Negative serum anti-transglutaminase (anti-tTG) and anti-endomysium (EmA) IgA antibodies.

(iii) Negative duodenal histology (absence of intestinal villous atrophy).

(iv) Negative IgE-mediated immuno-allergy tests to wheat (skin prick tests and serum-specific IgE-RASTs).

(v) Resolution of the symptoms on gluten-free diet and their reappearance on DBPC wheat challenge.

Additional inclusion criteria were: (i) age $>18$ years; (ii) follow-up duration longer than 8 months after the initial diagnosis; (iii) $>2$ outpatient visits during the follow-up period.

Exclusion criteria were: (i) IgA deficiency; (ii) self-exclusion of wheat from the diet; (iii) lack of DBPC-challenge method in the diagnosis.

\section{Diagnostic procedures}

In all cases, WS or multiple food hypersensitivity was diagnosed after the exclusion of other diagnoses. Consequently, the diagnostic workup of the patients may also have included abdominal ultrasonography, colonoscopy, histological examination of the recto-colon mucosa, small intestine barium examination, and $\mathrm{H} 2$ breath test.

\section{Immunologic assays and HLA typing for CD}

All patients underwent serum anti-tTG and EmA IgA, anti-gliadin (AGA) IgA and IgG assays performed using commercial kits (Eu.tTG. IgA, anti-Gliadin IgA and IgG, and anti-endomisio, Eurospital Pharma, Trieste, Italy), and reference values were determined as described previously $(8,9)$.

Most patients also underwent EmA assay in the culture medium of the intestinal biopsies $(10,11)$. Patients were also typed for
HLA-DQ phenotypes by PCR using sequence-specific primers, with a DR and DQ sequence-specific primers kit (Unipath SpA, Milan, Italy) or a rapid method (DQ-CD Typing Plus by BioDiaGene, Palermo, Italy) (12).

\section{Histology studies}

Duodenal histology. In accordance with a standard diagnostic protocol, four to six biopsy specimens were obtained from the second duodenal portion during gastroduodenoscopy. The slides were stained with hematoxylin and eosin and graded according to the standardized scheme reported by Oberhuber et al. (13). The number of intra-epithelial lymphocytes (IELs) per 100 villous epithelial cells was assessed by immunohistochemical staining: CD3 + IELs were stained with monoclonal antibody Leu-4. The upper limit of the reference interval in our laboratory was 25 IELs/100 epithelial cells. The number of eosinophils (EOS) per high-power field $(\times 40)$ was also assessed; the upper limit of the reference interval in our laboratory was $60 \mathrm{EOS} / 10$ high-power fields.

Other sites of histology examinations. In all the patients who underwent colonoscopy, biopsies were performed in the ileum and in different parts of the colon, according to a standard procedure (14). IELs and EOS were counted at a high-power field $(\times 40)$ in cross-sections of 50 crypts randomly selected from each slide and these cell numbers were expressed per 100 deep-crypt epithelial cells. The EOS count in the lamina propria was expressed as a percentage of EOS per 1,000 lamina propria cells per section (five sections per biopsy were examined). On the basis of the mean values +2 s.d. observed in the colon biopsies of the control subjects, the upper limits of the reference interval in our laboratory were: intra-epithelial lymphocytes $<7$, intra-epithelial EOS $<4$, and lamina propria $\mathrm{EOS}<9$.

\section{Allergology tests}

Specific (RAST) IgE and/or skin prick tests to food allergens, as well as serum anti- $\beta$ lactoglobulin IgG assays were performed in all patients, as previously described (15). Many patients underwent a flow cytometric basophil activation test, based on the demonstration of altered membrane phenotypes on allergen-activated basophils (16) (for details see Supplementary Information).

\section{Elimination diet and DBPC-challenge method}

At entry to the study, those patients who had self-reduced wheat consumption were invited to assume a minimum quantity of $30 \mathrm{~g}$ of wheat daily and were observed for 2-4 weeks on a regular diet. All patients then commenced a standard elimination diet, based on our previous experience $(14,16)$, with the exclusion of wheat, cow's milk, eggs, tomato, and chocolate. Patients selfreporting food hypersensitivity were also asked to avoid ingestion and/or contact with the food(s) causing symptoms. A previously validated questionnaire was used to assess self-reported food hypersensitivity (16).

Furthermore, food diaries were maintained by the patients during the above periods to assess dietary intake and adherence to the diet. 
After 4 weeks on elimination diet, they underwent DBPC challenges. The challenges were performed with the reintroduction of a single food at a time. Details about DBPC challenges are given in the Supplementary Information online. In the case of wheat, the DBPC challenge was performed with capsules coded A or B containing wheat or xylose, respectively. Capsules A or B were given for 2 consecutive weeks and then after 1 week of washout the patients received the other capsules for another 2 weeks (crossover design).

During all the phases of the study, including the challenge period, the severity of symptoms was recorded: the patients completed a $100-\mathrm{mm}$ visual analog scale, with 0 representing no symptoms, which assessed overall symptoms and the specific symptoms they each reported.

DBPC challenge for cow's milk proteins was performed with an identical method, according to a standard protocol $(15,16)$, at least 4 weeks before or after wheat challenge and when the visual analog scale (VAS) score was $<10$, on elimination diet.

The challenges were stopped when clinical reactions occurred (increase in VAS score $>30$ ) for at least two consecutive days (onset of abdominal discomfort or pain, associated with a change in stool frequency and/or appearance). The challenges were considered positive if the same symptoms, which had been initially presented, reappeared after their disappearance on elimination diet.

Challenges for other foods in patients with suspected multiple food hypersensitivity were performed in an open manner.

Two control groups were selected. One was composed of 50 patients with IBS who were classified as not suffering from wheat and food sensitivity, as they resulted negative at the DBPC challenges. These subjects were selected at random from those who underwent elimination diet and DBPC challenge during the same years.

The second control group was composed of $100 \mathrm{CD}$ patients showing gastrointestinal symptoms, selected at random from those diagnosed according to standard criteria (1) during the same years and in the same clinical units where the wheat-sensitive patients were diagnosed.

\section{Statistical analysis}

The sample size was calculated to compare the clinical, laboratory, and histological characteristics of the study groups. Choosing an error of 0.05 (alpha error) and, considering that the prevalence of gluten sensitivity has been reported to be about $6 \%$ in a tertiary care center for $\mathrm{CD}$ diagnosis (6), choosing a delta error of 0.03 , the number of patients to select will be given by the following calculation: $\left(1.96^{2} \times 0.06 \times 0.94\right) / 0.03^{2}=240(17)$.

When data distribution was Gaussian, values were expressed as mean \pm s.d. and differences between the two groups were calculated using the Student's $t$-test. Analysis of variance for unpaired data was used to compare data with a Gaussian distribution from more than two groups. When a significant difference was found, intergroup comparisons (post hoc analysis) were made with the Bonferroni test.

When data distribution was non-Gaussian, values were expressed as median (minimum-maximum) and differences between the two groups were calculated using the Mann-Whitney $U$-test. The Kruskal-Wallis one-way analysis of variance by ranks was used to compare data with a non-Gaussian distribution from more than two groups. When a significant difference was found, intergroup comparisons (post hoc analysis) were made with the MannWhitney $U$-test with a Bonferroni correction.

The analysis for pairwise data was performed using the Wilcoxon rank sum test, as the distribution of data was non-Gaussian.

The $\chi^{2}$ test or Fisher's exact test was used to test the frequencies.

SPSS software package (version 16.0; Chicago, IL) was used for statistical analysis.

The study was approved by the Ethics Committee of the University Hospital of Palermo.

\section{RESULTS}

During the study period, 920 patients with IBS diagnosis underwent the elimination diet and subsequent DBPC challenge (Figure 1); of these, 276 (30\%) were suffering from WS as they became asymptomatic (VAS score $<10$ ) on elimination diet and showed symptoms again (increase in VAS score $>30$ ) during the DBPC challenge.

Seventy patients were diagnosed with WS alone (group 1) and two hundred and six were diagnosed with multiple food hypersensitivity, including WS (group 2), as they also reacted to the DBPC challenge with cow's milk proteins. The patients suffering from WS alone (group 1) showed a clinical reaction to wheat challenge after a median time of 3 days (range: $3 \mathrm{~h}$ to 9 days), whereas those with multiple food hypersensitivity (group 2) reacted after a median of 2.5 days (range: $2 \mathrm{~h}$ to 5 days; Mann-Whitney; $P<0.01$ ). Ten patients in group 1 and thirty-two in group 2 did not complete the 2 -week challenge period due to the severity of symptoms, all when they were consuming wheat. No patients reacted to placebo.

Figure 2 shows the overall symptom score and the score for abdominal pain, bloating, and changes in stool consistency in the 276 patients suffering from WS, considered as a whole group. The VAS score for each symptom was significantly higher than at baseline (on the wheat-free diet) from the first week on the wheatcontaining diet $(P<0.0001$; Wilcoxon rank sum test $)$ and the values further increased at the end of the second week $(P<0.0001)$. On placebo, there was no significant variation in the VAS score over baseline after weeks 1 and 2. The score on the wheat-containing diet was significantly higher than on placebo both at the end of the first and second weeks into DBPC challenge $(P<0.0001$; Mann-Whitney).

None of the patients suffering from WS showed increased indexes of inflammation, such as serum C-reactive protein, erythrocyte sedimentation rate, or high white blood cell count, either before or after the challenges.

None of the patients suffering from WS alone (group 1) reacted to the DBPC challenge with cow's milk proteins and no other foods caused clinical symptoms. On the contrary, all patients with multiple food hypersensitivity (group 2) reacted to the DBPC cow's milk protein challenge: symptoms, time, and severity of the reaction were similar to those described for the wheat challenge. The absence of lactose ingestion during the challenge excluded 


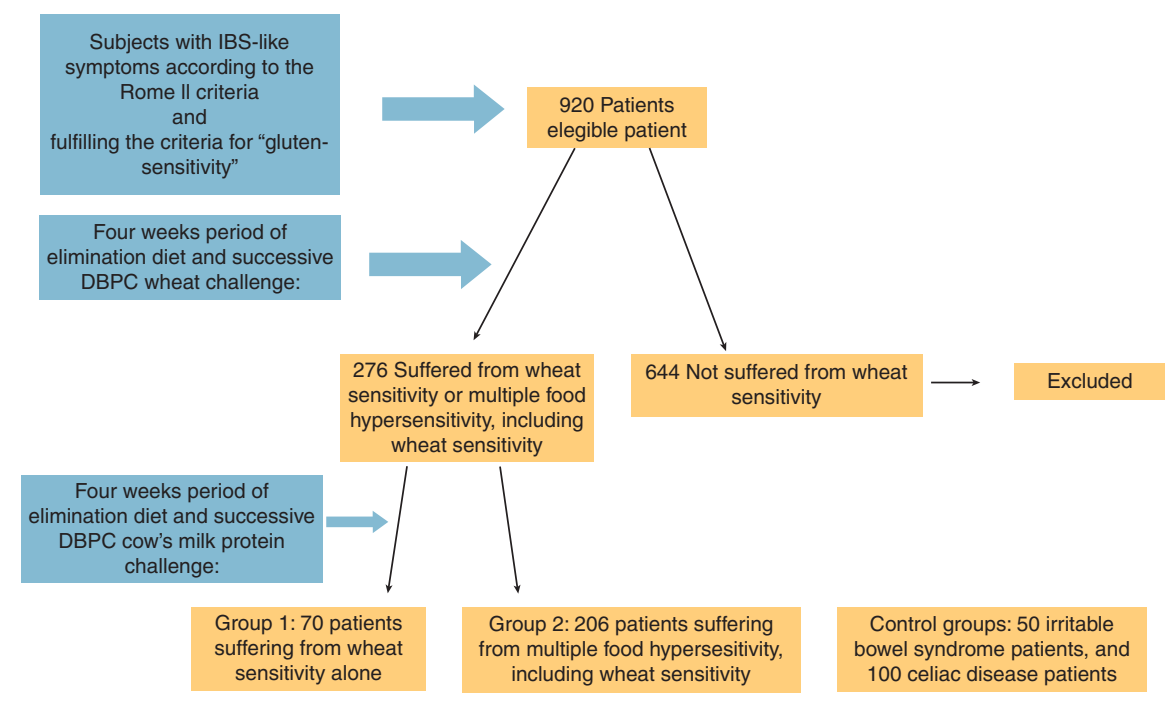

Figure 1. Study design. The clinical charts of 920 patients fulfiling the Rome II criteria for irritable bowel syndrome (IBS) diagnosis and the criteria for the diagnosis of "gluten sensitivity" (6) were revised. All patients underwent at least 4 weeks of elimination diet (exclusion of wheat, cow's milk and derivatives, eggs, tomato, and chocolate). Then they underwent double-blind placebo-controlled (DBPC) wheat challenge and 276 (30\%) reacted to the challenge. All these patients went back on the same elimination diet and after 4 weeks underwent DBPC cow's milk protein challenge. Seventy patients did not react to the challenge and subsequently re-introduced all the other foods - except wheat — without any problems: these patients composed group 1 (wheat-sensitive patients). Two hundred and six patients also reacted to cow's milk protein challenge: these patients composed group 2 (multiple food-sensitive patients).
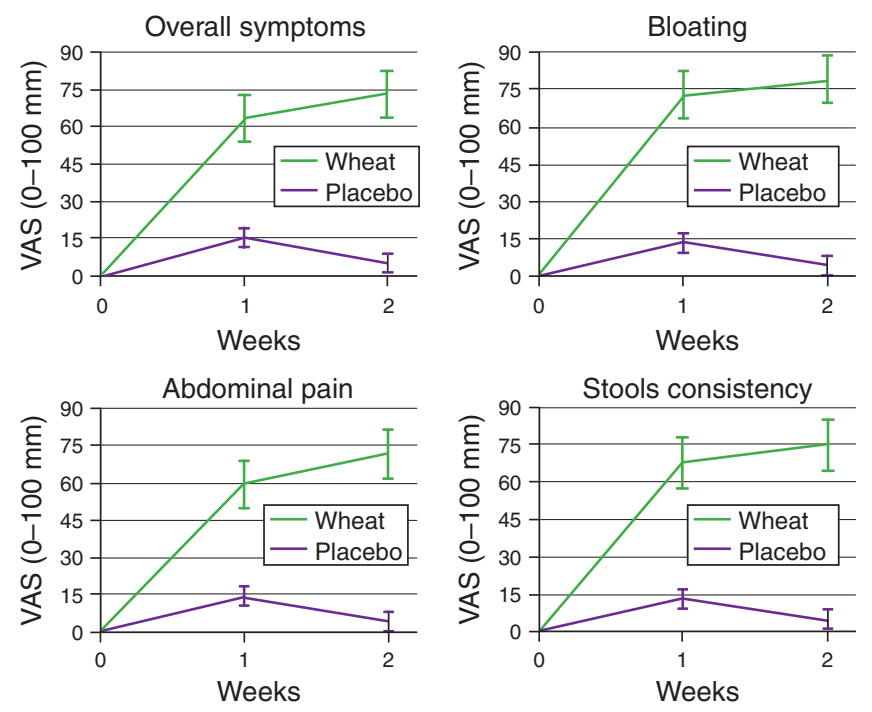

Figure 2. Changes in symptom severity over baseline during the doubleblind placebo-controlled wheat challenge: 2 weeks on wheat-containing diet and 2 weeks on placebo. Mean values + s.d. are given. VAS, visual analog scale.

the possibility that lactose intolerance could be responsible for the reappearance of symptoms. Furthermore, the patients in group 2 also showed symptoms when eating several foods, the most common being eggs (120 cases) and tomato (112 cases).

Table 1 summarizes the demographic and clinical characteristics of the two patient groups suffering from WS compared with the control groups of CD patients and IBS.
When we considered the 276 patients suffering from WS as a whole group, they showed an anemia (most sideropenic anemia) and weight loss frequency intermediate between the CD and IBS control groups and a higher frequency of coexistent atopic diseases, self-reported wheat intolerance, and history of food allergy in infancy than both the CD and IBS controls (Table 2). Furthermore, wheat-sensitive patients had undergone a higher number of previous gastrointestinal endoscopy examinations (esophagogastroduodenoscopy plus colonoscopy) than the CD and IBS controls $(P<0.001$ for both groups) during their lives.

Table 3 shows the results of the serum AGA, HLA typing, and immuno-allergy tests. The patients with WS showed a quite high frequency of positive serum IgG and IgA AGA. However, the allergy assay that tested positive most frequently was the cytometric basophil activation test. Among the wheat-sensitive patients, a small subgroup showed positive EmA assay in the culture medium of the duodenal biopsies.

Table 4 shows the duodenal and colon histology findings in the study groups. In accordance with the exclusion criteria, no patients suffering from WS showed intestinal villous atrophy, whereas all the CD patients showed various degrees of villous atrophy. There was a very high frequency of duodenal lymphocytosis in patients with WS and DQ2- and/or DQ8-positive patients showed a higher CD3 number than those DQ2/DQ8 negatives $(49+9$ vs. $40+6$ IEL/100EC; $t=9.6 ; P<0.0001)$. Furthermore, WS patients showed duodenal intra-epithelial EOS infiltrate. Lymphocytic inflammation of the colon mucosa was less frequent than in the duodenum in wheat-sensitive patients. On the contrary, EOS infiltration of the colonic mucosa characterized two-third of wheat-sensitive patients (Figure 3). In patients with multiple food hypersensitivity, the 
Table 1. Demographic and IBS-characteristics of the patients suffering from wheat sensitivity alone (group 1), from multiple food hypersen sitivity, including wheat, (group 2), from CD and from IBS

\begin{tabular}{|c|c|c|c|c|c|}
\hline & Group $1(n=70)$ & Group $2(n=206)$ & CD patients $(n=100)$ & IBS controls $(n=50)$ & $P$ value \\
\hline $\begin{array}{l}\text { IBS Type: Diarrhea/Constipation/ } \\
\text { Alternate bowel mov }\end{array}$ & $28 / 14 / 28(40 / 20 / 40 \%)$ & $86 / 51 / 69(42 / 25 / 33 \%)$ & $53 / 20 / 27(53 / 20 / 27 \%)$ & $16 / 14 / 20(32 / 28 / 40 \%)$ & NS \\
\hline
\end{tabular}

Table 2. Clinical characteristics of the patients suffering from WS ( $n=276), \mathrm{CD}(n=100)$, and IBS $(n=50)$

\begin{tabular}{|c|c|c|c|c|}
\hline & ws & CD & IBS & $P$ value \\
\hline Weight loss & $94 / 276(35 \%)$ & $52 / 100(52 \%)$ & $2 / 50(4 \%)$ & $\begin{array}{l}\text { CD vs. WS } 0.002 \\
\text { WS vs. IBS } 0.0001 \\
\text { CD vs. IBS } 0.0001\end{array}$ \\
\hline Median (range) number of previous endoscopies & $3.5(1-6)$ & $1(1-3)$ & $0.5(0-2)$ & $\begin{array}{c}\text { WS vs. CD and IBS } \\
0.001 \\
\text { CD vs. IBS NS }\end{array}$ \\
\hline Self-reported wheat intolerance & $137 / 276(50 \%)$ & $22 / 100(22 \%)$ & $7 / 50(14 \%)$ & $\begin{array}{c}\text { WS vs. CD and IBS } 0.0001 \\
\text { CD vs. IBS NS }\end{array}$ \\
\hline Coexistent atopic diseases & $80 / 276(29 \%)$ & $8 / 100(8 \%)$ & $3 / 50(6 \%)$ & $\begin{array}{c}\text { WS vs. } C D \text { and IBS } 0.0001 \\
\text { CD vs. IBS NS }\end{array}$ \\
\hline
\end{tabular}

severity of intra-epithelial EOS and lymphocytic infiltration gradually decreased from the ileum to the rectum (data not shown).

Histologic examination showed a uniformly normal crypt architecture, normal goblet cell number, and absence of granulomata or parasites.

Finally, Table 5 shows the clinical, serologic, and histologic characteristics that differed between patients with WS alone and those with multiple food sensitivity. Patients with WS alone (group 1), when compared with patients with multiple food sensitivity (group 2) showed a higher frequency of anemia and weight loss, and of the HLA haplotypes DQ2 or DQ8. EmA assay in the culture medium of the intestinal biopsies was positive in about one-third of the patients with WS alone, whereas none of the group 2 patients tested positive. Patients with multiple food sensitivity showed a higher frequency of coexistent atopic disease and self-reported wheat intolerance $(P<0.0001)$.

There was a significantly higher frequency of positive serum IgG anti-betalactoglobulin $(P<0.01)$ and of positive flow cytometric basophil activation tests $(P<0.0001)$ in the patients with multiple food hypersensitivity than in those with WS alone.

In patients with multiple food sensitivity, histology showed a significantly higher EOS infiltration of the duodenal and colonic mucosa than in patients with WS alone.

\section{DISCUSSION}

Although there is increasing recognition that gluten ingestion can also cause various disorders, mainly IBS-like ones outside the criteria for a CD diagnosis (4), very few studies have been performed in this field.

WS is frequently perceived by the patients themselves and they consult physicians seeking to reach a definite diagnosis of CD or "at least" of wheat hypersensitivity but this is generally opposed as the patients do not fulfill the CD diagnostic criteria (1) and do not show laboratory assays documenting an IgE-mediated food allergy. On the other hand, the emotional role is known to be pivotal in IBS patients $(18,19)$, consequently the clinical response 
Table 3. Number and percentage of positive test results for: serum AGA IgA and IgG, HLA haplotype DQ2 and/or DQ8, serum antibetalactoglobulin IgG, and flow cytometric basophil activation test in patients with WS and in the control groups (CD and IBS)

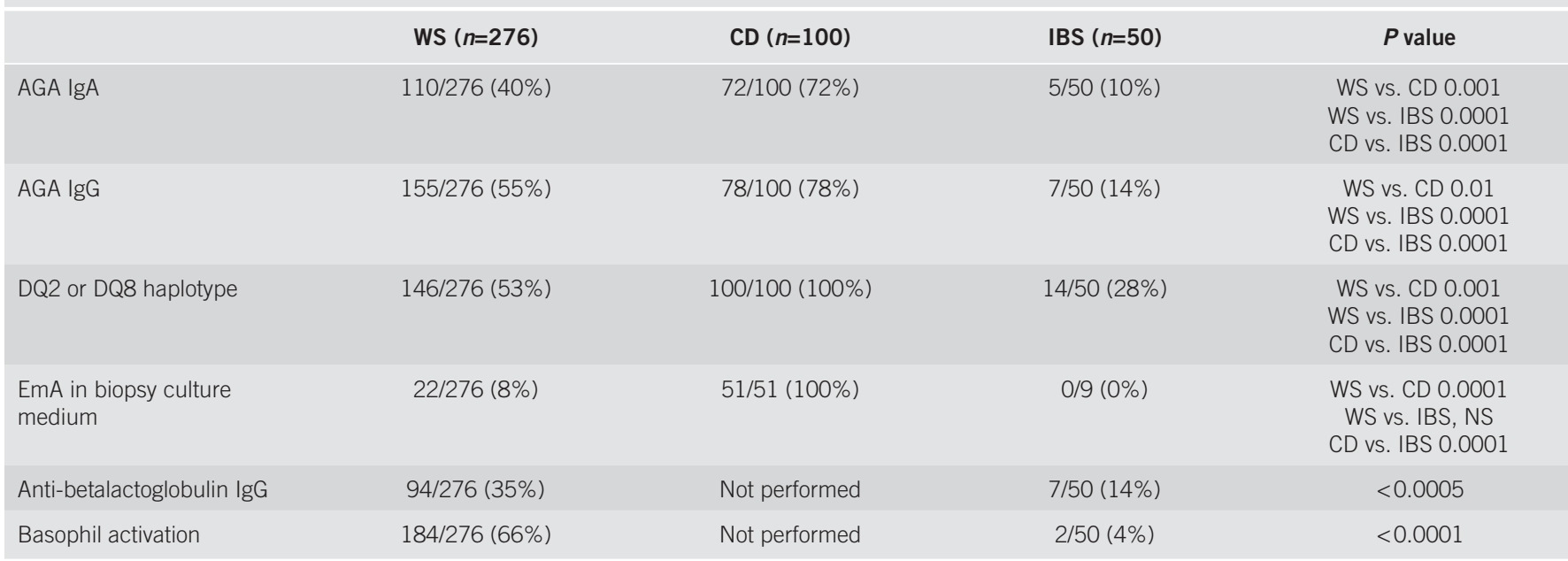

AGA, anti-gliadin; CD, celiac disease; IBS, irritable bowel syndrome; NS, not significant; WS, wheat sensitivity.

Notes: not all patients belonging to CD and IBS groups underwent EmA assay in the culture medium.

Table 4. Duodenal and colon histology findings in patients with WS and in the control groups (celiac disease-CD - and irritable bowel syndrome - IBS)

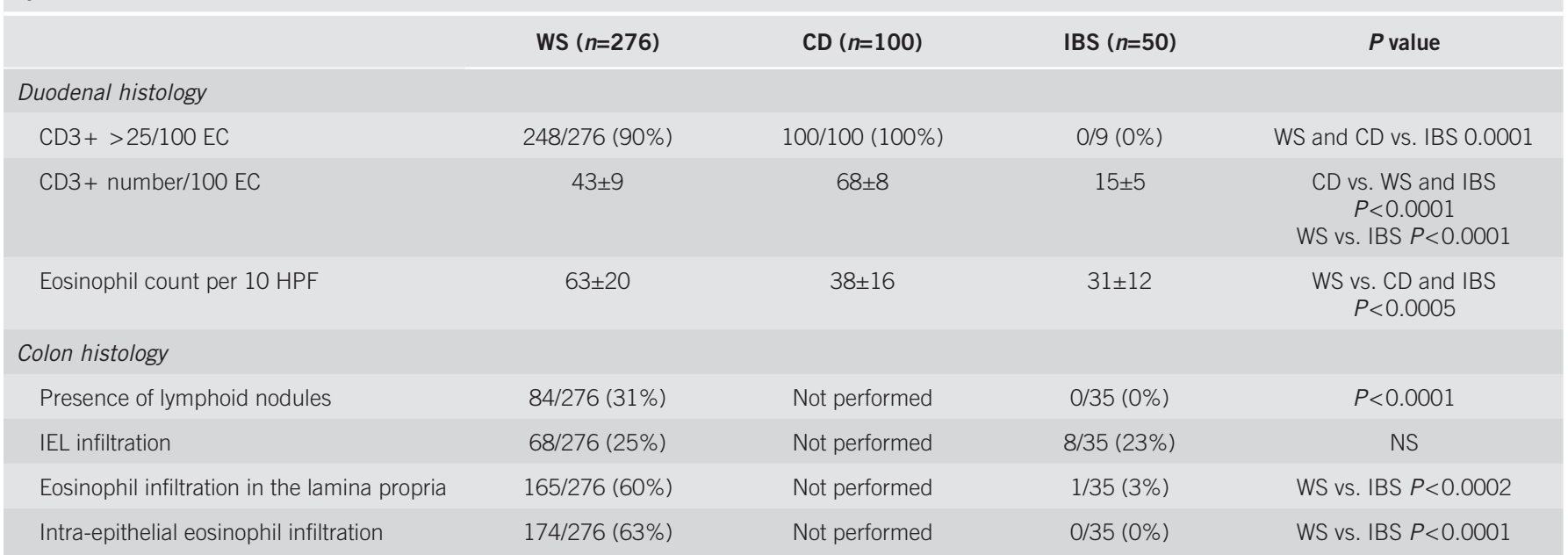

CD, celiac disease; EC, enterocytes; HPF, high-power field; IBS, irritable bowel syndrome; IEL, intra-epithelial lymphocytes; NS, not significant; WS, wheat sensitivity.

The numbers and percentages of patients showing the various histological findings are given.

Notes: Presence of lymphoid nodules indicates the number of patients showing this biopsy finding.

For the lymphocyte and eosinophil numbers the upper normal limit is indicated in the "Methods" section.

Not all IBS patients underwent upper and colon endoscopies: the exact number is given for each examination.

to an elimination diet and the use of the DBPC challenge to confirm the diagnosis of gluten hypersensitivity are suggested (20).

We have used the DBPC-challenge method in numerous studies on patients with suspected food hypersensitivity, as it is considered the gold standard for this diagnosis $(21,22)$. In this study, we reviewed the characteristics of a large group of IBS patients fulfiling the recent criteria proposed for "gluten sensitivity" who had a diagnosis of wheat hypersensitivity following DBPC challenge.

Our results clearly showed that a relevant percentage-more than one-fourth-of the patients who underwent DBPC wheat challenge were really suffering from WS.
As regards the clinical characteristics of WS patients and the identification of possible diagnostic markers, our data indicated that the presence of anemia and weight loss and a history of food allergy in infancy and of coexistent atopic diseases were more frequent in WS patients than in IBS controls. As other studies have not shown changes in intestinal permeability and absorption $(20,23)$, it could be hypothesized that the anemia and weight loss may in part depend on the self-restricted diet commenced by the patients, which excluded many foods. The other clinical characteristics are very similar to those observed in the allergic patients. Furthermore, the very high frequency of self-reported wheat intolerance, 


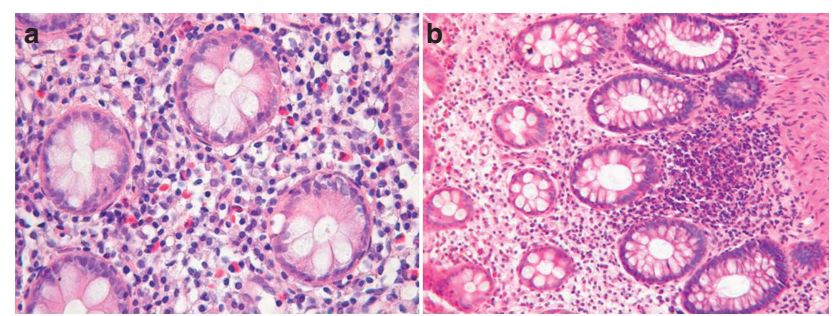

Figure 3. Histological evidence of significant eosinophil infiltration in the lamina propria of the mucosa of the right colon (a-hematoxylin and eosin (H\&E), 40) and lymphoid follicle hyperplasia (b-H\&E, 20) in a patient with multiple food hypersensitivity.

which we observed in our patients, should induce clinicians to pay full attention to patient suggestions.

As regards the serologic assays, we confirmed that about half of WS patients had positive AGAs $(20,24)$. More interestingly, the in vitro basophil activation assay confirmed a high sensitivity for WS diagnosis (16) and further studies would be very useful to confirm its use, as it seems to be the most accurate marker of WS at present.

Colon histology evaluation showed intra-epithelial and lamina propria infiltration of EOS in about two-third of cases and this finding was also frequently observed in the duodenum, together with lymphocytosis. This histology pattern could be another element pointing to a suspected WS diagnosis and the diffuse ileum-colon involvement could explain why the main symptoms in these patients were "lower" and not "upper" ones.

However, our data confirmed the idea that WS is a heterogeneous clinical condition, comprising various subgroups of patients who have different clinical histories and characteristics. In fact, we "identified" at least two distinct kinds of WS: one grouping patients with WS alone and another with subjects intolerant to wheat, cow's milk protein, and many other foods. These two groups, in fact, showed a clear variation in presentation and immunological findings, although there was also a significant overlap.

Subjects with WS alone showed a higher frequency of the DQ2 or DQ8 HLA haplotype, duodenal lymphocytosis was seen in $94 \%$ of cases and EmA assay in the culture medium of the duodenal biopsies tested positive in one-third of them. Although we found negative serum anti-tTG and EmA assays and an absence of villous atrophy, a previous study of ours had demonstrated that symptomatic patients who produce EmA in the duodenal culture can subsequently develop villous atrophy when remaining on a gluten-containing diet (12) and identical findings have been reported for serum EmA-positive patients with an initial evaluation of normal duodenal histology (25), as well as for patients without villous atrophy but with immunohistochemical evidence of anti-tTG deposits in the duodenal mucosa (26). These findings permitted us to hypothesize that a percentage of these patients could be predisposed to develop villous atrophy_-overt" CD—in the future. However, whether these patients will eventually develop CD remains to be examined in follow-up studies.

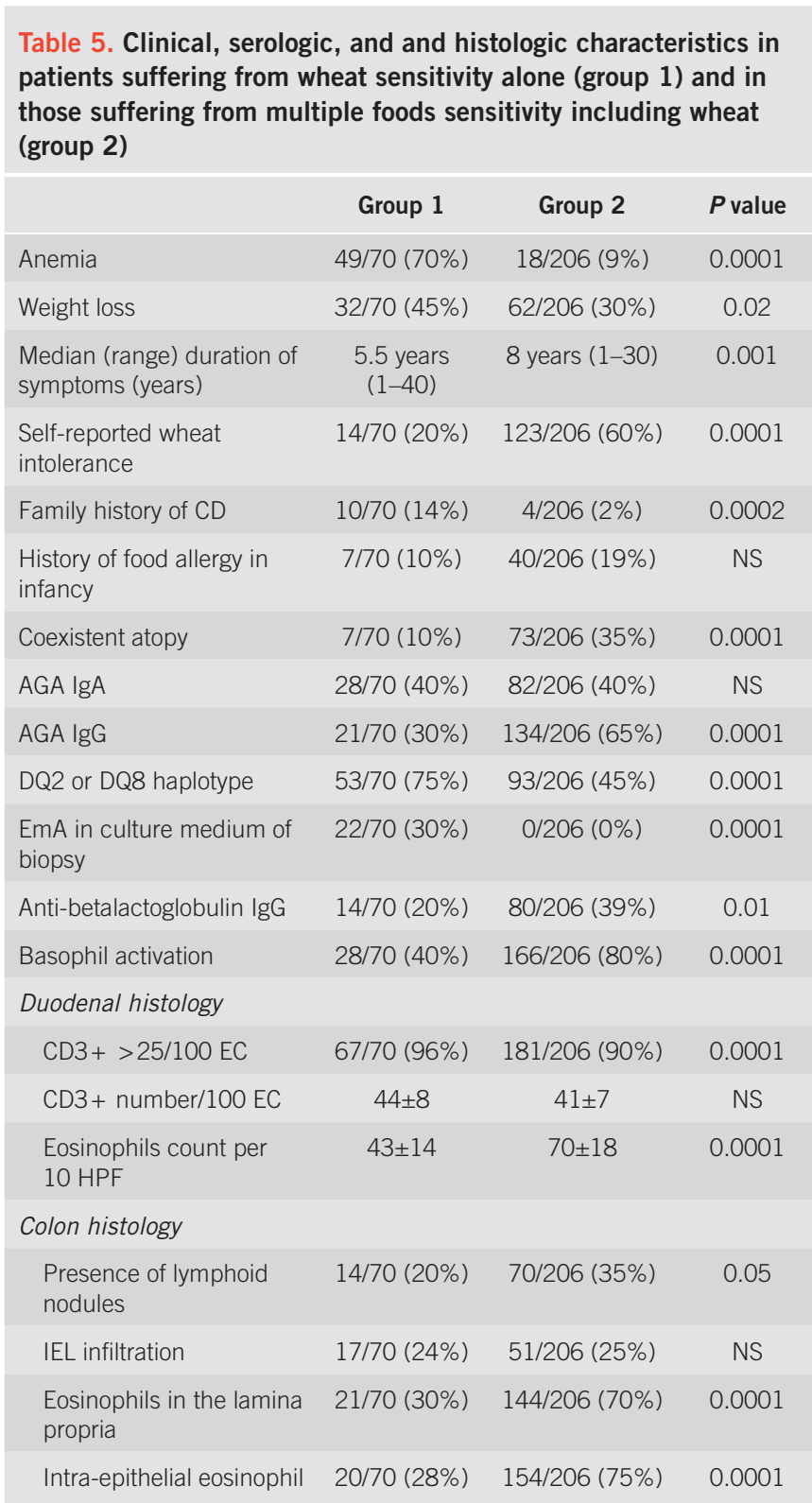

AGA, anti-gliadin; $C D$, celiac disease; EC, enterocytes; HPF, high-power field; NS, not significant.

Obviously, other hypotheses must be considered; experimental models have demonstrated that gluten sensitization of DQ8 mice increases acetylcholine release by the myenteric plexus and this can lead to consequent in vivo dysmotility (27). In this model, gluten did not cause villous atrophy, but there was evidence that coexistent triggers, e.g., intestine-damaging drugs or dysbacteriosis, can lead to a more severe intestinal impairment (28). Clearly, wheat antigens may also act in a similar manner.

The second group of patients with WS-those showing multiple food hypersensitivity-showed characteristics more similar to those of allergic rather than of $\mathrm{CD}$ patients, although none of them tested positive for IgE-based assays. In accordance with the "allergy hypothesis", these patients showed a higher frequency of 
family and personal history of food allergy and coexistent atopy than the other group. Their predominant presence in our study group probably conditioned the results of the immunology assays (positivity of IgG AGA and anti-betalactoglobulin, positivity of basophil activation assay) and of the histology studies (mucosal EOS infiltration in the duodenum and colon). Further studies are needed to clarify whether this predominant presence of allergiclike patients can be considered a "universal" characteristic of WS.

However, the limitations of our study must be underlined. This is a retrospective study and the data were not recorded according to a predesigned protocol. Although at least four duodenal biopsy samples were examined, we cannot exclude the possibility that a greater number of biopsy samples could have permitted a CD diagnosis in some cases (29). Furthermore, in most of these patients duodenal biopsy was not performed in the bulb and this has recently come to be considered a recommended biopsy site for $\mathrm{CD}$ diagnosis (30). Duodenal IEL infiltration can be caused by several factors $(31,32)$ and these causes were not all systematically examined. Our series is not composed of consecutive patients as we included only subjects who underwent DBPC challenge. This did not permit us to evaluate the respective incidences of the kinds of WS and could have determined a selection bias. As a point of reference, over the last year, taking into consideration both patients tested with open and DBPC challenge, we have diagnosed, in a single center, 46 new $\mathrm{CD}$ cases, 15 cases of wheat hypersensitivity alone, and 90 cases with multiple food hypersensitivity. Furthermore, in this study we did not use gluten but wheat for the challenges. Consequently, we cannot exclude the possibility that other components of wheat may be responsible for the pictures we describe: i.e., fructans and poorly absorbed carbohydrates can induce symptoms by themselves (33).

In conclusion, our data clearly identified a patient population suffering from non-CD WS and described their clinical, serologi$\mathrm{cal}$, and histologic characteristics. We also suggest the possibility of distinct populations of subjects with WS: one with characteristics more similar to $\mathrm{CD}$ and the other with characteristics strongly pointing to food allergy.

\section{ACKNOWLEDGMENTS}

We thank Ms Carole Greenall for her precious help in revising the English. We also thank other physicians who recruited and followed up the patients during the study period, in particular Dr Lidia Di Prima.

\section{CONFLICT OF INTEREST}

Guarantor of the article: Antonio Carroccio, MD.

Specific author contributions: Study design, patient recruitment and follow-up, data collection and interpretation, and writing the manuscript: Antonio Carroccio; patient recruitment and follow-up, data collection and interpretation, and revising the manuscript: Pasquale Mansueto; data interpretation and revising the manuscript: Giuseppe Iacono and Giovanni B Rini; histology study, data collection and interpretation: Ada M Florena; patient recruitment and follow-up, data collection and interpretation: Alberto D'Alcamo, Francesca Cavataio, Aurelio Seidita, and Giuseppe Pirrone; immunology assays, data collection, and interpretation: Ignazio Brusca; statistics, data collection, and interpretation: Maurizio Soresi; patient recruitment and follow-up, data collection: Giuseppe Ambrosiano.

Financial support: This study was supported by a Grant from the University of Palermo (MIUR-Ricerca Scientifica di Ateneo, ex quota $60 \%$, years 2006-7).

Potential competing interests: None.

\section{Study Highlights}

\section{WHAT IS CURRENT KNOWLEDGE}

It has been reported that about $6 \%$ of people referred to a tertiary center for celiac disease are suffering from "gluten sensitivity", but the real existence of this condition is still debated.

$\checkmark$ Many patients suffering from IBS-like symptoms self-report a "gluten sensitivity" and their symptoms improve on a gluten-free diet.

There are no markers of gluten sensitivity (GS) and only one study to date, including only a small number of patients, has confirmed the existence of GS as a defined clinical condition, using the diagnostic gold standard-the doubleblind placebo-controlled food challenge.

\section{WHAT IS NEW HERE}

Our results clearly showed that a relevant percentage-onethird-of our irritable bowel syndrome patients who underwent DBPC wheat challenge were really suffering from WS.

WS patients were characterized by frequent self-reported wheat intolerance and coexistent atopy and food allergy in infancy.

The main histological characteristic of WS patients was eosinophil infiltration of the duodenal and colon mucosa.

We suggest that WS is a heterogeneous condition, which includes different subgroups of patients. In particular, we found that the patients suffering from WS alone were characterized by clinical features more similar to those found in CD patients; in contrast, patients suffering from multiple food sensitivity were characterized by clinical features similar to those found in allergic patients.

\section{REFERENCES}

1. Walker-Smith JA, Guandalini S, Schmitz J et al. Revised criteria for diagnosis of coeliac disease. Arch Dis Child 1990;65:909-11.

2. Mäki M, Mustalahti K, Kokkonen J et al. Prevalence of celiac disease among children in Finland. N Engl J Med 2003;348:2517-24.

3. Green PH, Cellier C. Celiac disease. N Engl J Med 2007;357:1731-43.

4. Verdu EF, Armstrong D, Murray JA. Between celiac disease and irritable bowel syndrome: the no man's land of gluten sensitivity. Am J Gastroenterol 2009;104:1587-94.

5. Troncone R, Jabri B. Celiac disease and gluten-sensitivity. J Intern Med 2011;269:582-90

6. Sapone A, Bai J, Ciacci C et al. Spectrum of gluten-related disorders: consensus on new nomenclature and classification. BMC Med 2012;10:13.

7. Thompson WG, Longstreth GF, Drossman DA et al. Functional bowel disorders and functional abdominal pain. Gut 1999;45 (Suppl. 2): 43-7.

8. Carroccio A, Vitale G, Di Prima L et al. Comparison of anti-transglutaminase ELISAs and anti-endomysial antibody assay in the diagnosis of celiac disease: a prospective study. Clin Chem 2002;48:1546-50.

9. Carroccio A, Iacono G, Montalto G et al. Immunologic and absorptive tests in celiac disease: can they replace intestinal biopsies? Scand J Gastroenterol 1993;28:673-6.

10. Picarelli A, Maiuri L, Frate A et al. Production of antiendomysial antibodies after in-vitro gliadin challenge of small intestine biopsy samples from patients with coeliac disease. Lancet 1996;348:1065-7. 
11. Carroccio A, Di Prima L, Pirrone G et al. Anti-transglutaminase antibody assay of the culture medium of intestinal biopsy specimens can improve the accuracy of celiac disease diagnosis. Clin Chem 2006;52:1175-80.

12. Carroccio A, Iacono G, Di Prima L et al. Antiendomysium antibodies assay in the culture medium of intestinal mucosa: an accurate method for celiac disease diagnosis. Eur J Gastroenterol Hepatol 2011;23:1018-23.

13. Oberhuber G, Granditsch G, Vogelsang H. The histopathology of coeliac disease: time for a standardized report scheme for pathologists. Eur J Gastroenterol Hepatol 1999;11:1185-94.

14. Iacono G, Ravelli A, Di Prima L et al. Colonic lymphoid nodular hyperplasia in children: relationship to food hypersensitivity. Clin Gastroenterol Hepatol 2007;5:361-6.

15. Iacono G, Cavataio F, Montalto G et al. Intolerance of cow's milk and chronic constipation in children. N Engl J Med 1998;338:1100-4.

16. Carroccio A, Brusca I, Mansueto P et al. A cytological assay for diagnosis of food hypersensitivity in patients with irritable bowel syndrome. Clin Gastroenterol Hepatol 2010;8:254-60.

17. Cochran WG. Sampling Techniques, 3rd edn John Wiley: New York, p 428.

18. Drossman DA. Review article: an integrated approach to the irritable bowel syndrome. Alim Pharmacol Ther 1999;13:3-14.

19. Lu CL, Chang FY. Placebo effect in patients with irritable bowel syndrome. J Gastroenterol Hepatol 2011;26 (Suppl. 3): 116-8.

20. Biesiekierski JR, Newnham ED, Irving PM et al. Gluten causes gastrointestinal symptoms in subjects without celiac disease: a double-blind randomized placebo-controlled trial. Am J Gastroenterol 2011;106:508-14.

21. Sampson HA, Sicherer SH, Birnbaum AH. AGA technical review on the evaluation of food allergy in gastrointestinal disorders. American Gastroenterological Association. Gastroenterology 2001;120:1026-40.

22. Boyce JA, Assa'ad A, Burks AW et al. Guidelines for the diagnosis and management of food allergy in the United States: report of the
NIAID-sponsored expert panel. J All Clin Immunol 2010;126 (Suppl. 6): S1-S58.

23. Sapone A, Lammers KM, Mazzarella G et al. Differential mucosal IL-17 expression in two gliadin-induced disorders: gluten sensitivity and the autoimmune enteropathy celiac disease. Int Arch Allergy Immunol 2010;152:75-80.

24. Volta U, Tovoli F, Cigola R et al. Serological tests in gluten-sensitivity. J Clin Gastroenterol; published online 5 December 2011 [e-pub ahead of print].

25. Kurppa K, Collin P, Viljamaa M et al. Diagnosing mild enteropathy celiac disease: a randomized, controlled clinical study. Gastroenterology 2009;136:816-23.

26. Salmi TT, Collin P, Korponay-Szabo IR et al. Endomysial antibody-negative coeliac disease: clinical characteristics and intestinal autoantibody deposits. Gut 2006;55:1746-53.

27. Verdu EF, Huang XX, Natividad J et al. Gliadin-dependent neuromuscular and epithelial secretory responses in the gut. Am J Physiol Gastrointest Liver Physiol 2008;294:G217-25.

28. Natividad JM, Huang X, Slack E et al. Host responses to intestinal microbial antigens in gluten-sensitive mice. PLoS One 2009;4:e6472.

29. Lebwohl B, Kapel RC, Neugut AI et al. Adherence to biopsy guidelines increases celiac disease diagnosis. Gastrointest Endosc 2011;74:103-9.

30. Evans KE, Aziz I, Cross SS et al. A prospective study of duodenal bulb biopsy in newly diagnosed and established adult celiac disease. Am J Gastroenterol 2011;106:1837-42.

31. Aziz I, Evans KE, Hopper AD et al. A prospective study into the etiology of lymphocytic duodenosis. Alim Pharmacol Ther 2010;32:1392-7.

32. Vande Voort JL, Murray JA, Lahr BD et al. Lymphocytic duodenosis and the spectrum of celiac disease. Am J Gastroenterol 2009;104:142-8.

33. Shepherd SJ, Parker FC, Muir JG et al. Dietary triggers of abdominal symptoms in patients with irritable bowel syndrome: randomized placebocontrolled evidence. Clin Gastroenterol Hepatol 2008;6:765-71. 